\title{
Ankle exercise with functional electrical stimulation affects spasticity and balance in stroke patients
}

\author{
Sun-Young Ha', Jun-Ho Han', Young Jun $\mathrm{Ko}^{2}$, Yun-Hee Sung ${ }^{1,3 *}$ \\ 'Department of Physical Therapy, Graduate School, Kyungnam University, Changwon, Korea \\ ${ }^{2}$ Major in Sport Service Practice, College of Welfare Covergence, Kangnam University, Yongin, Korea \\ ${ }^{3}$ Department of Physical Therapy, College of Health Sciences, Kyungnam University, Changwon, Korea
}

Stroke patients have limited motor function due to ankle spasticity, and various interventions are applied to solve this problem. The purpose of this study was to investigate the effects of functional electrical stimulation (FES) with ankle exercise on spinal cord motor neuron excitability and balance in stroke patients. Twenty-five stroke patients were divided into the three groups. For the intervention, the control group applied general physiotherapy, the experimental group I applied a sham FES with ankle exercise, and the experimental group II applied a FES with ankle exercise. All groups applied the intervention for 30 min per session, 5 times a week, for a total of 8 weeks. The functional reaching test
(FRT), Timed Up and Go test was used to measure balance ability, and $\mathrm{H}$-reflex was used to measure spinal motor neuron excitability. All tests were measured before and after the intervention. In the ankle exercise with FES group, spinal motor neuron excitability significantly decreased $(P<0.05)$, and FRT was significantly increased $(P<0.05)$. Therefore, FES with ankle exercise for stroke patients could be suggested as an effective intervention for improving motor function.

Keywords: Ankle exercise, Functional electrical stimulation, Stroke, H-reflex

\section{INTRODUCTION}

Stroke is a central nervous system disorder associated with motor impairment, perception, and cognitive impairment due to ischemic or hemorrhagic damage to the brain (Dijkerman et al., 2004). Stroke patients are accompanied by problems such as muscle weakness, abnormal muscle tone and movement patterns, poor balance, and fine motor function (Chen and Patten, 2006; Eng and Tang, 2007). This leads to a decrease in joint movement, joint contracture, and foot drop which makes walking difficult due to reduced action of the ankle dorsiflexor muscle (Chung et al., 2004; Park et al., 2020).

Most stroke patients have a spasticity in at least one joint. In particular, the spasticity of the lower extremities causes abnormal muscle tone due to cocontraction of the agonist and antagonist muscles during walking (Lamontagne et al., 2000; Lamontagne et al., 2001). Approximately $76 \%$ of stroke patients are accompa-

nied by spasticity of the ankle joint (Inghilleri et al., 2003). Plantarflexion spasticity of the ankle joint is a major cause of poststroke disability, and acts as a factor that decreases the walking ability of stroke patients (Lamontagne et al., 2000; Lamontagne et al., 2001). Ankle joint spasticity is caused by disinhibition of alpha-motor neurons and gamma motor neurons in the anterior horn of the spinal cord due to damage to the upper motor neuron. Due to this, the spinal motor neuron excitability is increased (Inghilleri et al., 2003). Budini et al. (2018) reported that passive lengthening movements of the ankle plantar flexor muscles decreased $\mathrm{H}$-reflex, which was indicated by presynaptic inhibition.

The ankle joint is the first posture control used to maintain balance, and plays an important role in restoring balance (Menz et al., 2005). The movement of ankle joint is mainly used when there is little sway on a firm surface, and it can maintain a standing balance through contraction of the muscles around the ankle (Almeida et al., 2006; Park et al., 2016). Stroke patients show ab-

${ }^{*}$ Corresponding author: Yun-Hee Sung (D) https://orcid.org/0000-0002-4877-9784 Department of Physical Therapy, College of Health Sciences, Kyungnam University, 7 Kyungnamdaehak-ro, Masanhappo-gu, Changwon 51767, Korea

E-mail: sungpt97@kyungnam.ac.kr

Received: October 26, 2020 / Accepted: November 22, 2020 
normal balance ability due to reduced weight transfer to paretic side and spasticity of the ankle joint, which reduces walking ability (Geiger et al., 2001). A consideration of ankle joint spasticity during intervention is an important factor for functional recovery of stroke patients. Many studies have shown that ankle exercise and functional electrical stimulation (FES) are effective in improving balance in stroke patients (Kim et al., 2015; Park et al., 2016; Sabut et al., 2011).

FES is widely used in clinical practice to solve ankle joint problems in stroke patients. This induces muscle contraction to the paralyzed muscle, creating a functionally useful motion. It is effective in preventing muscular atrophy and improving dysfunction (Kapadia et al., 2014). In particular, applying FES with specific tasks in stroke patients can promote the overall condition of the muscles and maximize the therapeutic effect (Castro et al., 2000). Many researchers reported that FES with exercise or only FES is effective for gait, balance, and muscles activity of the lower limb in stroke patients (Aaron et al., 2018; Hwang et al., 2015; Zheng et al., 2018). However, studies on the effect of these intervention on spasticity, a major problem in stroke patients, are insufficient. Therefore, we determined the effects of ankle exercise with FES on the spasticity and balance in stroke patients.

\section{MATERIALS AND METHODS}

\section{Participants}

This study was conducted for stroke patients admitted to Taebong hospital (Chanwon, Korea). Inclusion criteria were (a) those who were diagnosed with cerebral infarction by computed tomography or magnetic resonance imaging, (b) those who had a disease period of 6 months or more after the onset of stroke, (c) those who had a score of 24 or more in Korean version of MiniMental State Examination, (d) those who can maintain an independent standing position for more than 1 minute without assistive devices or help, (e) A person whose passive range of motion of ankle joint becomes neutral position. Exclusion criteria were (a) those with congenital anomalies and orthopedic diseases in the lower extremities, (b) those who have unilateral neglect, or have difficulty concentrating or remembering, (c) those who are ineligible for FES treatment (ex, artificial pacemaker). Those who satisfy the above conditions were selected, all participants were those who signed the consent form after listening the purpose and procedure of the study. It was conducted after approval of the institutional review board of Kyungnam University (1040460-A-2015017).
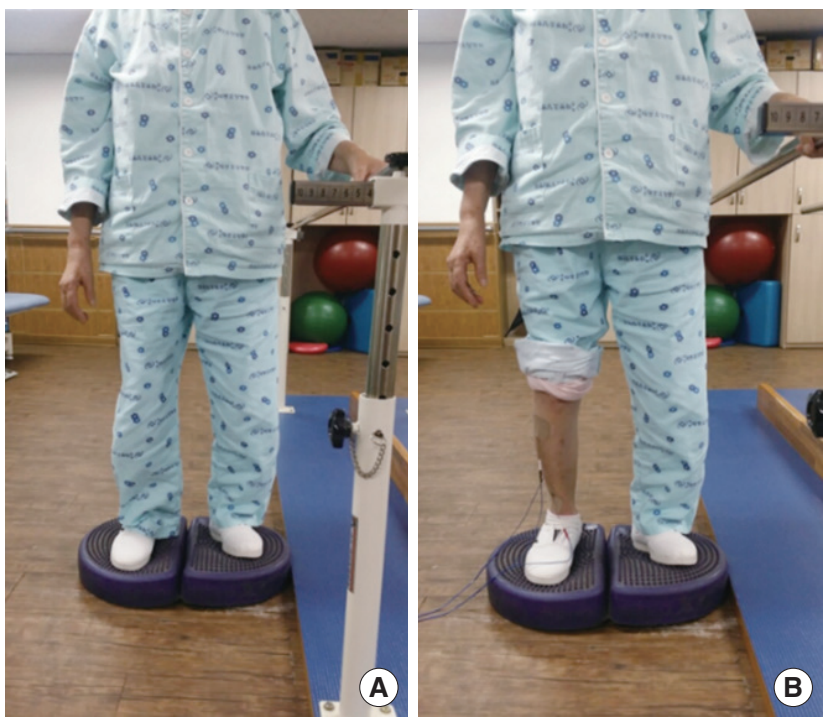

Fig. 1. Intervention. (A) Ankle exercise on aero-step. (B) Ankle exercise with functional electrical stimulation on aero-step.

The participants were randomly assigned to the control group, experimental group I, and experimental group II. In the control group applied general physical therapy, and experimental group I applied a sham FES with ankle exercise, and experimental group II applied a FES with ankle exercise (Fig. 1). All groups applied the intervention for $30 \mathrm{~min}$ per session, 5 times a week, for a total of 8 weeks, and the $\mathrm{H}$-reflex, balance was examined equally before and after the intervention (Fig. 2).

\section{Ankle exercise}

The ankle exercise was performed in hard form and in aero-step, respectively, and the contents are shown in Table 1.

\section{Functional electrical stimulation}

FES (Microstim Model GmbH, Stanberg, Germany) was simultaneously applied agonist and antagonist (i.e., tibialis anterior muscle and gastrocnemius muscle) of paraplegic ankle joint (Sabut et al., 2011). The waveform of the FES was applied as a symmetrical abnormal wave, an amplitude of $250 \mu \mathrm{sec}$, a frequency of $40 \mathrm{~Hz}$, and an intensity of 20 to $40 \mathrm{~mA}$.

\section{Spinal cord motor neuron excitatory}

Hoffman reflex (H-reflex) is a monosynaptic segmental reflex used to evaluate the functional state of the reflex arch at the spinal cord level (Christie et al., 2004). Surface electromyography (MP150, BIOPAC Systems, Gelato, CA, USA) was used to measure spinal motor neuron excitatory. The sampling rate of the electromyogra- 


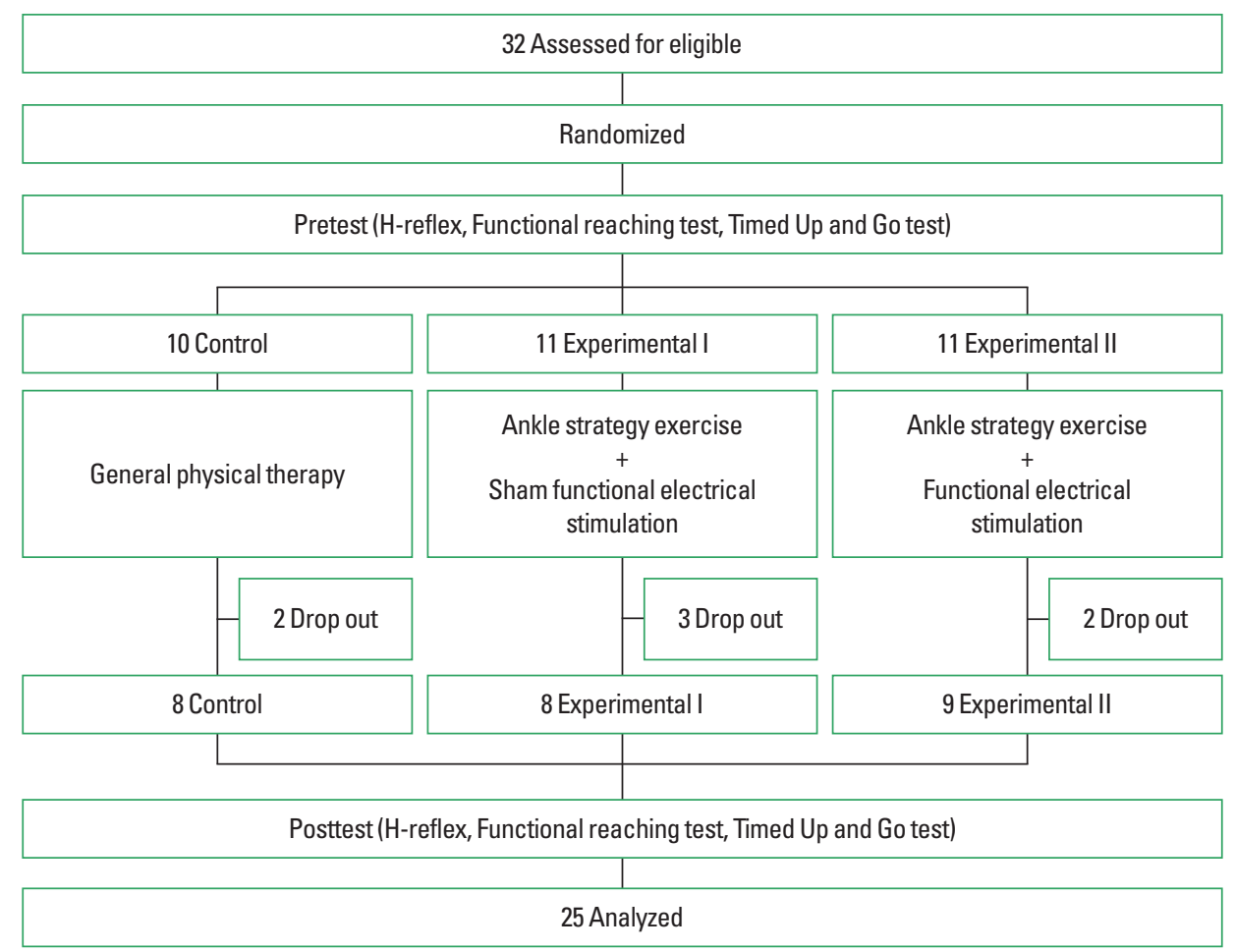

Fig. 2. Flow chart.

Table 1. Ankle exercise

\begin{tabular}{lll}
\hline Exercise & \multicolumn{1}{c}{ Frequency } & \multicolumn{1}{c}{ Exercise program (1 set) } \\
\hline Exercise on hard floor & $\begin{array}{l}\text { 1,2 Sets with eyes open } \\
\text { 3 Sets of eyes closed }\end{array}$ & - Lifting and lowering the heels of both feet \\
& $\begin{array}{l}\text { 20 Times for each item } \\
\text { Exercise on aero-step }\end{array}$ & - Lifting and lowering the front of both feet \\
& $\begin{array}{l}\text { 1, } 2 \text { Sets with eyes open (Apply exercise by holding the } \\
\text { arm support with one hand) 20 times for each item }\end{array}$ & $\begin{array}{l}\text { - Rotate your torso left and right in a standing position with your feet at shoulder width } \\
\text { - Moving weight from left to right in kneeling position }\end{array}$ \\
\hline
\end{tabular}

phy (EMG) signal was set to $1,000 \mathrm{~Hz}$, and the amplified waveform was set to a band pass filter of 30 to $500 \mathrm{~Hz}$, and a notch filter of $60 \mathrm{~Hz}$ was used to remove noise from the EMG signal. Data were analyzed with commercially-available software (AcqKnowledge, BIOPAC Systems, Goleta, CA, USA). The test-retest reliability is $r=0.97$ (Handcock et al., 2001).

The patient placed his ankle out of the bed to relax the calf muscles in a prone position, and the ankle was placed in the neutral position. The recording electrode was attached to the belly of medial soleus muscle, and the reference electrode was attached to the distal $15 \mathrm{~cm}$ along the Achilles tendon. The stimulating electrode stimulated the tibial nerve in the popliteal region by placing the negative electrode in the proximal part, and the ground electrode was attached between the stimulating electrode and the recording electrode (Palmieri et al., 2004). In order to reduce muscle resis- tance, the hair was removed using a disposable razor on the area to be attached to the electrode, and then the skin was cleaned with a rubbing alcohol cotton, and the electrode was attached. When electrical stimulation is applied to the peripheral nerves, M-wave appears to the muscles directly through the efferent motor neuron from the stimulated point. H-reflex appears through the spinal cord and alpha-motor neuron through the afferent fibers from the stimulated point (Kohan et al., 2010). The amplitude ratio (H/M ratio) of $\mathrm{H}$-reflex to the maximum $\mathrm{M}$-wave is used as an index to objectively evaluate spasticity by reflecting the excitability of motor neurons (Kramer et al., 2013).

\section{Functional reaching test}

Functional reaching test (FRT) was taped to a shoulder-high wall, and participant placed $5 \mathrm{~cm}$ away from the wall and stood 
side by side. With both elbows stretched and the shoulder joint at a $90^{\circ}$ position, the arm should be extended forward as much as possible without bending the knee or hip joint. At this time, the moving length of the tip of the third finger was recorded, and the average value was used by measuring 3 times. The interrater reliability is $r=0.98$, and the interrater reliability is $r=0.98$ (MerchánBaeza et al., 2014).

\section{Timed Up and Go test}

Timed Up and Go (TUG) test is a method to measure the time from sitting in a chair with armrests to walking up to $3 \mathrm{~m}$ and then returning to a chair. The average value was used by measuring 3 times using a stopwatch. The interrater reliability is $r=0.98$, and the intra-rater reliability is $r=0.99$ ( $\mathrm{Ng}$ and HuiChan, 2005).

\section{Statistical analysis}

For statistical analysis, IBM SPSS Statistics ver. 20.0 (IBM Co., Armonk, NY, USA) was used. Shapiro-Wilk test was performed for normal distribution, and one-way analysis of variance was used to compare the differences (pre-post) between groups. Duncan test was used for post hoc analysis. All statistical significance levels $\alpha$

Table 2. Medical characteristics of participants ( $N=25)$

\begin{tabular}{lcccc}
\hline Characteristic & $\begin{array}{c}\text { Control } \\
(\mathrm{n}=8)\end{array}$ & $\begin{array}{c}\text { Experimental I } \\
(\mathrm{n}=8)\end{array}$ & $\begin{array}{c}\text { Experimental II } \\
(\mathrm{n}=9)\end{array}$ & P-value \\
\hline Paralysis, left:right & $5: 3$ & $2: 6$ & $6: 3$ & \\
Onset period (mo) & $44.3 \pm 28.8$ & $26.9 \pm 25.1$ & $27.0 \pm 28.4$ & 0.391 \\
MMSE-K (score) & $27.1 \pm 2.3$ & $25.4 \pm 1.5$ & $26.3 \pm 2.8$ & 0.333 \\
Brunnstrom stage (stage) & $5.4 \pm 0.5$ & $4.9 \pm 0.6$ & $5.0 \pm 0.9$ & 0.356 \\
\hline
\end{tabular}

Values are presented as mean \pm standard deviation.

MMSE-K, Korean version of Mini-Mental State Examination. were below 0.05 .

\section{RESULTS}

\section{Medical characteristics of participants}

The medical characteristics of the participants are shown in Table 2 , and there was no statistically significant difference between the three groups $(P>0.05)$ (Table 2$)$.

\section{Comparison of spinal motor neuron excitability}

In the difference of the H-reflex, the experimental group II was significant difference from the experimental group I and control $(P<0.05)$, but there was no significant difference between the experimental group I and control $(P>0.05)$. There was significant difference between the experimental group II and control $(P<0.05)$. In the difference of the $\mathrm{M}$-wave, there was no significant difference between the three groups $(P>0.05)$. In the difference of the H/M ratio, the experimental group II was significant difference from the control group and the experimental group I $(P<0.05)$, but there was no significant difference between the experimental group I and control $(P>0.05)$ (Table 3).

\section{Comparison of balance}

In the difference of the FRT, there was significant difference between the experimental group II and the control group $(P<0.05)$. In the difference of the TUG, the experimental group I and the experimental group II were significant difference from the control group $(P<0.05)$, but there was not significant difference between experimental group I and the experimental group II $(P>0.05)$ (Table 4).

Table 3. Comparison of spinal motor neuron excitability

\begin{tabular}{|c|c|c|c|c|c|c|}
\hline Variable & Group & Pre & Post & Pre-Post & $P$-value & Duncan \\
\hline \multirow[t]{3}{*}{ H-reflex (mV) } & Control $^{\mathrm{a})}$ & $1.22 \pm 1.26$ & $3.08 \pm 1.05$ & $-1.85 \pm 0.45$ & $0.001^{*}$ & $a=b<c$ \\
\hline & Experimental $\left.\right|^{\mathrm{b})}$ & $1.36 \pm 1.06$ & $4.22 \pm 1.59$ & $-2.86 \pm 1.51$ & & \\
\hline & Experimental II ${ }^{c}$ & $2.99 \pm 1.67$ & $3.47 \pm 1.37$ & $-0.48 \pm 0.99^{*}$ & & \\
\hline \multirow[t]{3}{*}{ M-wave (mV) } & Control $^{\text {a) }}$ & $5.07 \pm 1.07$ & $10.35 \pm 2.87$ & $-5.28 \pm 3.11$ & 0.721 & $a=b=c$ \\
\hline & Experimental I ${ }^{\mathrm{b})}$ & $4.37 \pm 2.61$ & $10.54 \pm 3.16$ & $-6.17 \pm 3.45$ & & \\
\hline & Experimental I|c) & $5.29 \pm 2.90$ & $3.47 \pm 1.37$ & $0.48 \pm 0.99$ & & \\
\hline \multirow[t]{3}{*}{$\mathrm{H} / \mathrm{M}$ ratio (\%) } & Controla) $^{a)}$ & $24.62 \pm 19.45$ & $33.01 \pm 17.16$ & $-8.39 \pm 15.22$ & $0.013^{*}$ & $a=b<c$ \\
\hline & Experimental I' & $29.60 \pm 15.30$ & $39.20 \pm 13.22$ & $-9.59 \pm 13.92$ & & \\
\hline & Experimental ||c) & $63.08 \pm 38.02$ & $41.18 \pm 22.16$ & $21.90 \pm 34.09^{*}$ & & \\
\hline
\end{tabular}

Values are presented as mean \pm standard deviation.

$\mathrm{H} / \mathrm{M}$ ratio, amplitude ratio.

${ }^{*} P<0.05$ compared to the control group. 
Table 4. Comparison of FRT and TUG

\begin{tabular}{|c|c|c|c|c|c|c|}
\hline Variable & Group & Pre & Post & Pre-Post & $P$-value & Duncan \\
\hline \multirow[t]{3}{*}{ FRT (cm) } & Control $^{\mid a)}$ & $5.95 \pm 2.80$ & $6.53 \pm 2.61$ & $-0.58 \pm 0.90$ & $0.024^{*}$ & $a=b<c$ \\
\hline & Experimental $\left.\right|^{b)}$ & $7.96 \pm 4.44$ & $8.87 \pm 4.73$ & $-0.91 \pm 0.35$ & & \\
\hline & Experimental $\|^{c)}$ & $13.35 \pm 9.34$ & $14.94 \pm 9.37$ & $-1.58 \pm 0.74^{*}$ & & \\
\hline \multirow[t]{3}{*}{ TUG (sec) } & Contro|al & $23.02 \pm 8.94$ & $22.18 \pm 9.12$ & $0.84 \pm 1.07$ & $0.023^{*}$ & $a<b=c$ \\
\hline & Experimental $\mathrm{I}^{\mathrm{b})}$ & $38.10 \pm 24.88$ & $35.17 \pm 23.30$ & $2.93 \pm 1.72^{*}$ & & \\
\hline & Experimental II) & $24.48 \pm 11.67$ & $21.95 \pm 10.80$ & $2.52 \pm 1.55^{*}$ & & \\
\hline
\end{tabular}

Values are presented as mean \pm standard deviation.

FRT, functional reach test; TUG, Timed Up and Go test.

${ }^{*} P<0.05$ compared to the control group.

\section{DISCUSSION}

The clinical significance of Hoffmann's reflex is an objective evaluation of spasticity using the amplitude ratio (H/M ratio) of H-reflex to the maximum M-wave. Patients with spasticity have poor neuromodulation ability compared to healthy people, resulting in an increased H/M ratio (Pizzi et al., 2005). Therefore, decline of excitability in the spinal motor neuron can be considered as a decrease in spasticity. In addition, $\mathrm{H} / \mathrm{M}$ ratio can be used as an objective indicator to evaluate the effect on diagnosis or treatment for spasticity. Chan et al. (2012) showed that the H/M ratio was significantly decreased after applying whole-body vibration in stroke patients. Barzi and Zehr (2008) found that applying arm cycling to stroke patients suppressed $\mathrm{H}$-reflex and was effective in improving motor function. Lo et al. (2009) reported that when applying short-term propulsion of a FES-assisted leg-cycling wheelchair to stroke patients, the H/M ratio decreased, indicating a decrease in spasticity. In this study, the $\mathrm{H} / \mathrm{M}$ ratio was significantly decreased after ankle exercise with FES. The reduction of $\mathrm{H} / \mathrm{M}$ ratio is consistent with the reduction of spasticity. Through these results, it is thought that ankle exercise with FES will decrease spinal motor neuron excitability and have a positive effect on reduction of spasticity.

The ankle movements are related to maintaining balance during walking. In addition, the ankle is essential for balance, as it regulates the interaction between the foot and the ground (Vlutters et al., 2019). Jeon and Choi (2015) stated that the ankle exercise was effective in improving balance in hemiplegic patients. Ruiz and Richardson (2005) mentioned that ankle exercise was effective in improving balance ability in elderly women. In addition, applying FES with specific tasks can promote the overall condition of the muscles and maximize the therapeutic effect (Castro et al., 2000). FES applied to the damaged lower limb provides afferent stimulation through repetitive exercise. In addition, it can have a positive effect on the recovery of motor function and stimulate the reorganization of neuromuscular activity by strengthening the connection network between sensory cortex, parietal lobe, and motor cortex (Classen et al., 1998; Lo et al., 2009). Hwang et al. (2015) reported that treadmill training with tilt sensor FES was more effective in improving balance ability than treadmill training with placebo tilt sensor FES in stroke patients. Zheng et al. (2018) reported that the FES in the lower extremities improved the balance ability more than the sham FES in stroke patients. The results of this study also showed that the FRT was improved in the ankle exercise with FES group than the ankle exercise with sham FES group. Therefore, it would be more effective to improve the balance ability of stroke patients if applying ankle exercise with FES. However, in the TUG, there was not significant difference in the ankle exercise with FES group and the ankle exercise with sham FES group. TUG is a tool for evaluating balance ability and predicting the risk of falls, testing functional mobility (Whitney et al., 2004). Therefore, the results of this study are suggested that TUG was difficult to improve the function of the participants during the 8-week intervention period because it included not only the balance but also the walking factor.

In conclusion, ankle exercise with FES was effective for the spinal motor neuron excitability, balance, and in stroke patients. Therefore, ankle exercise with FES could be suggested as an intervention for improving the function of stroke patients. The limitation of this study is that it is difficult to generalize the results of the study with a small number of participants. Therefore, in the future studies, studies that supplement these limitations should be conducted.

\section{CONFLICT OF INTEREST}

No potential conflict of interest relevant to this article was reported. 


\section{REFERENCES}

Aaron SE, Vanderwerker CJ, Embry AE, Newton JH, Lee SCK, Gregory CM. FES-assisted cycling improves aerobic capacity and locomotor function postcerebrovascular accident. Med Sci Sports Exerc 2018;50: 400-406.

Almeida GL, Carvalho RL, Talis VL. Postural strategy to keep balance on the seesaw. Gait Posture 2006;23:17-21.

Barzi Y, Zehr EP. Rhythmic arm cycling suppresses hyperactive soleus H-reflex amplitude after stroke. Clin Neurophysiol 2008;119:1443-1452.

Budini F, Christova M, Gallasch E, Rafolt D, Tilp M. Soleus H-reflex inhibition decreases during $30 \mathrm{~s}$ static stretching of plantar flexors, showing two recovery steps. Front Physiol 2018;9:935.

Castro MJ, Apple DF Jr, Rogers S, Dudley GA. Influence of complete spinal cord injury on skeletal muscle mechanics within the first 6 months of injury. Eur J Appl Physiol 2000;81:128-131.

Chan KS, Liu CW, Chen TW, Weng MC, Huang MH, Chen CH. Effects of a single session of whole body vibration on ankle plantarflexion spasticity and gait performance in patients with chronic stroke: a randomized controlled trial. Clin Rehabil 2012;26:1087-1095.

Chen G, Patten C. Treadmill training with harness support: selection of parameters for individuals with poststroke hemiparesis. J Rehabil Res Dev 2006;43:485-498.

Christie A, Lester S, LaPierre D, Gabriel DA. Reliability of a new measure of H-reflex excitability. Clin Neurophysiol 2004;115:116-123.

Chung SG, Van Rey E, Bai Z, Roth EJ, Zhang LQ. Biomechanic changes in passive properties of hemiplegic ankles with spastic hypertonia. Arch Phys Med Rehabil 2004;85:1638-1646.

Classen J, Liepert J, Wise SP, Hallett M, Cohen LG. Rapid plasticity of human cortical movement representation induced by practice. J Neurophysiol 1998;79:1117-1123.

Dijkerman HC, Ietswaart M, Johnston M, MacWalter RS. Does motor imagery training improve hand function in chronic stroke patients? A pilot study. Clin Rehabil 2004;18:538-549.

Eng JJ, Tang PF. Gait training strategies to optimize walking ability in people with stroke: a synthesis of the evidence. Expert Rev Neurother 2007;7:1417-1436

Geiger RA, Allen JB, O'Keefe J, Hicks RR. Balance and mobility following stroke: effects of physical therapy interventions with and without biofeedback/forceplate training. Phys Ther 2001;81:995-1005.

Handcock PJ, Williams LR, Sullivan SJ. The reliability of H-reflex recordings in standing subjects. Electromyogr Clin Neurophysiol 2001;41:915.

Hwang DY, Lee HJ, Lee GC, Lee SM. Treadmill training with tilt sensor functional electrical stimulation for improving balance, gait, and mus- cle architecture of tibialis anterior of survivors with chronic stroke: a randomized controlled trial. Technol Health Care 2015;23:443-452.

Inghilleri M, Lorenzano C, Conte A, Frasca V, Manfredi M, Berardelli A. Effects of transcranial magnetic stimulation on the $\mathrm{H}$ reflex and $\mathrm{F}$ wave in the hand muscles. Clin Neurophysiol 2003;114:1096-1101.

Jeon SN, Choi JH. The effects of ankle joint strategy exercises with and without visual feedback on the dynamic balance of stroke patients. J Phys Ther Sci 2015;27:2515-2518.

Kapadia NM, Nagai MK, Zivanovic V, Bernstein J, Woodhouse J, Rumney P, Popovic MR. Functional electrical stimulation therapy for recovery of reaching and grasping in severe chronic pediatric stroke patients. J Child Neurol 2014;29:493-499.

Kim K, Lee S, Kim D, Kim KS. The effects of ankle joint muscle strengthening and proprioceptive exercise programs accompanied by functional electrical stimulation on stroke patients' balance. J Phys Ther Sci 2015;27:2971-2975.

Kohan AH, Abootalebi S, Khoshnevisan A, Rahgozar M. Comparison of modified ashworth scale and hoffmann reflex in study of spasticity. Acta Med Iran 2010;48:154-157.

Kramer A, Gollhofer A, Ritzmann R. Acute exposure to microgravity does not influence the H-reflex with or without whole body vibration and does not cause vibration-specific changes in muscular activity. J Electromyogr Kinesiol 2013;23:872-878.

Lamontagne A, Malouin F, Richards CL. Contribution of passive stiffness to ankle plantar flexor moment during gait after stroke. Arch Phys Med Rehabil 2000;81:351-358.

Lamontagne A, Malouin F, Richards CL. Locomotor-specific measure of spasticity of plantar flexor muscles after stroke. Arch Phys Med Rehabil 2001;82:1696-1704.

Lo HC, Tsai KH, Su FC, Chang GL, Yeh CY. Effects of a functional electrical stimulation-assisted leg-cycling wheelchair on reducing spasticity of patients after stroke. J Rehabil Med 2009;41:242-246.

Menz HB, Morris ME, Lord SR. Foot and ankle characteristics associated with impaired balance and functional ability in older people. J Gerontol A Biol Sci Med Sci 2005;60:1546-1552

Merchán-Baeza JA, González-Sánchez M, Cuesta-Vargas AI. Reliability in the parameterization of the functional reach test in elderly stroke patients: a pilot study. Biomed Res Int 2014;2014:637671.

$\mathrm{Ng}$ SS, Hui-Chan CW. The timed up \& go test: its reliability and association with lower-limb impairments and locomotor capacities in people with chronic stroke. Arch Phys Med Rehabil 2005;86:1641-1647.

Palmieri RM, Ingersoll CD, Hoffman MA. The hoffmann reflex: methodologic considerations and applications for use in sports medicine and athletic training research. J Athl Train 2004;39:268-277.

Park KH, Lim JY, Kim TH. The effects of ankle strategy exercises on un- 
stable surfaces on dynamic balance and changes in the COP. J Phys Ther Sci 2016;28:456-459.

Park SJ, Kim TH, Oh S. Immediate effects of tibialis anterior and calf muscle taping on center of pressure excursion in chronic stroke patients: a cross-over study. Int J Environ Res Public Health 2020;17:4109.

Pizzi A, Carlucci G, Falsini C, Verdesca S, Grippo A. Evaluation of upperlimb spasticity after stroke: a clinical and neurophysiologic study. Arch Phys Med Rehabil 2005;86:410-415.

Ruiz R, Richardson M. Functional balance training using a domed device. Strength Cond J 2005;27:50-55.

Sabut SK, Sikdar C, Kumar R, Mahadevappa M. Improvement of gait \& muscle strength with functional electrical stimulation in sub-acute \& chronic stroke patients. Annu Int Conf IEEE Eng Med Biol Soc 2011;
2011:2085-2088.

Vlutters M, van Asseldonk EHF, van der Kooij H. Ankle muscle responses during perturbed walking with blocked ankle joints. J Neurophysiol 2019;121:1711-1717.

Whitney SL, Marchetti GF, Schade A, Wrisley DM. The sensitivity and specificity of the Timed "Up \& Go" and the Dynamic Gait Index for self-reported falls in persons with vestibular disorders. J Vestib Res 2004;14:397-409.

Zheng X, Chen D, Yan T, Jin D, Zhuang Z, Tan Z, Wu W. A randomized clinical trial of a functional electrical stimulation mimic to gait promotes motor recovery and brain remodeling in acute stroke. Behav Neurol 2018;2018:8923520. 